\title{
Modeling of cell sorting and rare cell capture with microfabricated biodevices
}

\author{
Q1 Gabor Jarvas, and Andras Guttman, ${ }^{1,2}$ \\ Q2 ${ }^{1}$ MTA-PE Translational Glycomics Group, University of Pannonia, Veszprem, Hungary \\ ${ }^{2}$ Barnett Institute, Northeastern University, Boston, MA, USA
}

In this paper, we review different aspects of computer modeling and simulation of lab-on-a-chip type bioanalytical devices, with special emphasis on cell sorting and rare cell capture, such as circulating tumor cells (CTCs). We critically review important fundamental concepts and innovative applications in addition to detailed analysis by multiphysics approaches. Relevant essentials of hydrodynamic, Newtonian, and non-Newtonian rheological behavior, single and multiphase models, together with various force field-mediated flows are discussed with respect to cell sorting. Furthermore, we provide a summary of techniques used to simulate electric and magnetic field-based rare cell capture methods, such as electrophoresis and magnetophoresis. Finally, we present simulations of practical applications to help non-specialists understand the basic principles and applications.

\section{Introduction}

Microfabricated biodevices (MBDs) are inspired by the electrical circuits of the semiconductor industry where all required components are integrated into a microchip in order to improve efficiency and also reduce operation cost and time. Analogously, MBDs (also known as lab-on-achip systems) can comprise micro-operation units such as miniaturized reactors, microseparation units, affinity capture chambers, storage compartments, etc., with a final goal of system integration. They are used for processing and analyzing minute amounts of biological fluids or other biological samples, such as cells [1-3].

During the past decade, MBDs entered the rapidly growing field of cell sorting. Rare cell capture is an important application in clinical diagnostic and biomedical research. Conventional cell sorting techniques are based on changes in the conductivity of micropores when a cell crosses them, optical detection of cells encapsulated at

Corresponding author: Guttman, A. (A.Guttman@neu.edu).

Keywords: cell sorting; cell capture; lab-on-a-chip; modeling; simulation; CFD.

0167-7799/\$ - see front matter

(c) 2013 Elsevier Ltd. All rights reserved. http://dx.doi.org/10.1016/j.tibtech.2013.10.001 high speed in droplets passing in front of a detector (flow cytometry), and cytology based on direct observation of cells spread on a slide or centrifuged onto it [1]. The major drawbacks of these techniques are the requirement for presorting (filtering, centrifugation, and rinsing), long sorting time, and the requirement for large sample volumes. Last but not least these devices need highly trained service personnel. Traditional pre-sorting methods, furthermore, can damage cells due to mechanical stress and could affect normal-life functionality. To avoid the above mentioned issues, fluorescence-activated cell sorting (FACS) [4], dielectrophoretic sorting [5], electrokinetic isolation, inertial separation, controlled pressure sorting [6,7], and magnetic-activated particle-based [8] methods have been proposed.

Cell sorting has particular importance in cancer research because the affected cells represent an extremely heterogenic system, where the reduction of complexity is of high necessity (for a review, see [9]). Furthermore, most metastases are thought to arise from cells that escape from the primary tumor and then transiently circulate in the cardiovascular system as CTCs [10]. Although very important in basic research and clinical diagnostics, the detection and capture of these cells is a great challenge because the typical number of CTCs in the blood range from one (if any) CTC per $10 \mathrm{ml}$ up to several hundreds of CTCs per $\mathrm{ml}[8,11]$. Biomarkers on the cancer cell surface or inside the cell are not abundant either [12]. Dealing with such very small amounts of samples and targets makes MBDs promising tools for detection, capture, and enrichment because the geometrical dimensions of both the targets and the working channels are in the same range.

Although modeling and simulation of microfluidic systems is primarily considered as a design tool, it can also be used to support experimental data interpretation [13]. In general, modeling is a complementary engineering tool to quickly achieve an optimal design at low cost with a minimum number of actual experiments. Furthermore, modeling holds the promise of custom-made applicationspecific solutions. With regard to $\mathrm{MBDs}$, computational fluid dynamics (CFD) modeling is widely accepted and probably the most used tool today. In this paper, we focus on the use of CFD-based simulations and critically review modeling methods for cell sorting for rare cell capture with MBDs. 


\section{The engineering aspects of microfluidics-based cell manipulation}

Microfluidics deals with manipulation of very small amounts of fluids $\left(10^{-9}-10^{-10} \mathrm{dm}^{3}\right)$. Typical dimensions of a microfluidic channel system range from a few to several hundred $\mu \mathrm{m}$, with wafer materials usually consisting of glass or various polymers [14]. The very high surface/ volume ratio and the typically parabolic (laminar) fluidic flow profile (term will be discussed later) are also important features of MBDs. The former plays an essential role, among others, in surface interaction-based techniques, such as sorting and affinity capture [15]. Due to geometric principles, the smaller the dimensions of the channels, the higher their surface/volume ratio. MBDs require only a very small amount of samples and reagents, feature rapid processing times, and provide high resolution separations, good detection sensitivity, and accuracy. The laminar flow characteristics enable miscible fluids to flow next to each other without turbulent mixing and without the necessity for physically separating the flows [16]. Concomitantly, in microchannels, the mass transport between parallel-flowing fluids occurs mainly by diffusion [17].

In laminar flow, the trajectory of any particle is not randomly dependent on the time element; the trace can be calculated if the boundary conditions are time invariant. As a consequence, convective transport, that is, fluidic flowmediated mass transport, occurs only in the direction of the flow, which can be characterized by a dimensionless parameter called the Reynolds number (Re), representing the ratio of viscous and inertial forces. Most often, the $R e$ is defined as:

$R e=\frac{\rho l v}{\mu}$

where $\rho$ is the density $\left(\mathrm{kg} / \mathrm{m}^{3}\right), l$ is the characteristic linear dimension $(\mathrm{m}), v$ is the mean velocity $(\mathrm{m} / \mathrm{s})$, and $\mu$ is the dynamic viscosity ( $\mathrm{Pa} \mathrm{s}$ ) of the fluid. Flows tend to be turbulent around $R e>2000$, but in certain instances can be laminar even at much higher Re numbers [18]. As an example, in a 100-micron-high channel, the flow of water at a typical speed of $0.01 \mathrm{~m} / \mathrm{s}$ has an Re of unity [19], which means that the flow is strongly laminar, that is, different layers of the fluid flow are parallel with each other and the wall of the channel. This feature of laminar flow can be used to separate diffusing compounds in adjacent fluid flows in a channel, as recently described in [16], where the authors developed a microfluidic cartridge for extraction of fluorescein from a mixture of fluorescein and dextran. The major portion of the dextran $(98.6 \%)$ was retained, whereas $43.1 \%$ of fluorescein was removed during one cycle. A CFD simulation was used to optimize extraction performance and microfluidic parameters.

\section{Pressure-driven flow models for cell sorting}

Physical phenomena are usually described by partial differential equations (PDEs), which can be solved either analytically or numerically. Analytical solutions are not always available, and numerical methods require an additional step called discretization (also known as meshing). According to the discretization methods of the governing equations, which are the essential foundation of different

\section{Box 1. Basic steps of CFD modeling}

1. Geometry design uses either built-in CFD program tools or computer-aided design (CAD) software such as AutoCAD or Invertor.

2. Specification of governing equations defines the form and associated coefficients in the governing partial differential equation, the boundary conditions, and the initial values.

3. Meshing splits the complex geometry of the modeled domain into smaller, primitive subdomains in order to solve the governing equations at each nodal point of the subdomains.

4. Solving uses different available algorithms depending on the nature of the problem for steady state or time-dependent studies.

5. Post-processing is the step where the calculated data is visualized by graphs, plots, and animations according to the problem at hand.

techniques for numerical modeling of fluid flow, two main groups can be identified as follows. One widely accepted technique includes finite difference, finite volume, and finite element methods, whereas the second group uses such infrequent methods as boundary element, spectral element, and other high resolution approaches. A comprehensive analysis of the major advantages and disadvantages of these techniques is published in [20]. There are numerous commercially available software packages, which are suitable for modeling fluidic flows in MBDs, such as Fluent, Ansys, CFD-ACE+, Flow3D, COMSOL Multiphysics, as well as free codes like OpenFVM or FreeFEM. Despite this diversity of software implementations, the basic steps are always the same, that is, design of geometry, definition of governing equations, meshing, solving, and post-processing (Box 1).

Figure 1A-D depicts the different stages of model building and examples of the resulting analysis. Most of the codes can solve very complex problems, even those involving challenging geometries. The solutions of PDEs can be considered, however, the results need to be examined carefully because the solutions could converge onto local minima showing unrealistic results, which could mislead an untrained user. To avoid such incorrect interpretations, simulations should be performed many times with different meshing methods and sizes to obtain grid-independent data.

Because laminar fluid flow is dominant in MBDs, CFD calculations are less complicated in the case of turbulent flow, and while time- and resource-consuming, the results obtained are often very reliable [21]. One of the major challenges is that narrow-bore channels have large aspect ratios, usually more than an order of magnitude (ratio of diameter and length) that makes meshing complex and requires the use of position-dependent discretization. Models can be constructed in one, two, or three dimensions and could be stationary or dynamic, that is, time independent and time dependent, respectively. The hierarchical modeling concept [22], also referred to as bottom-up design, considers molecular to whole system levels and could be adapted to CFD modeling. One of the practical ways is to start the simulation with a simplified model and then improve towards more complex stages. For example, the calculated stationary flow profile (frequently referred to as the flow field) could be the starting point for a dynamic model. 


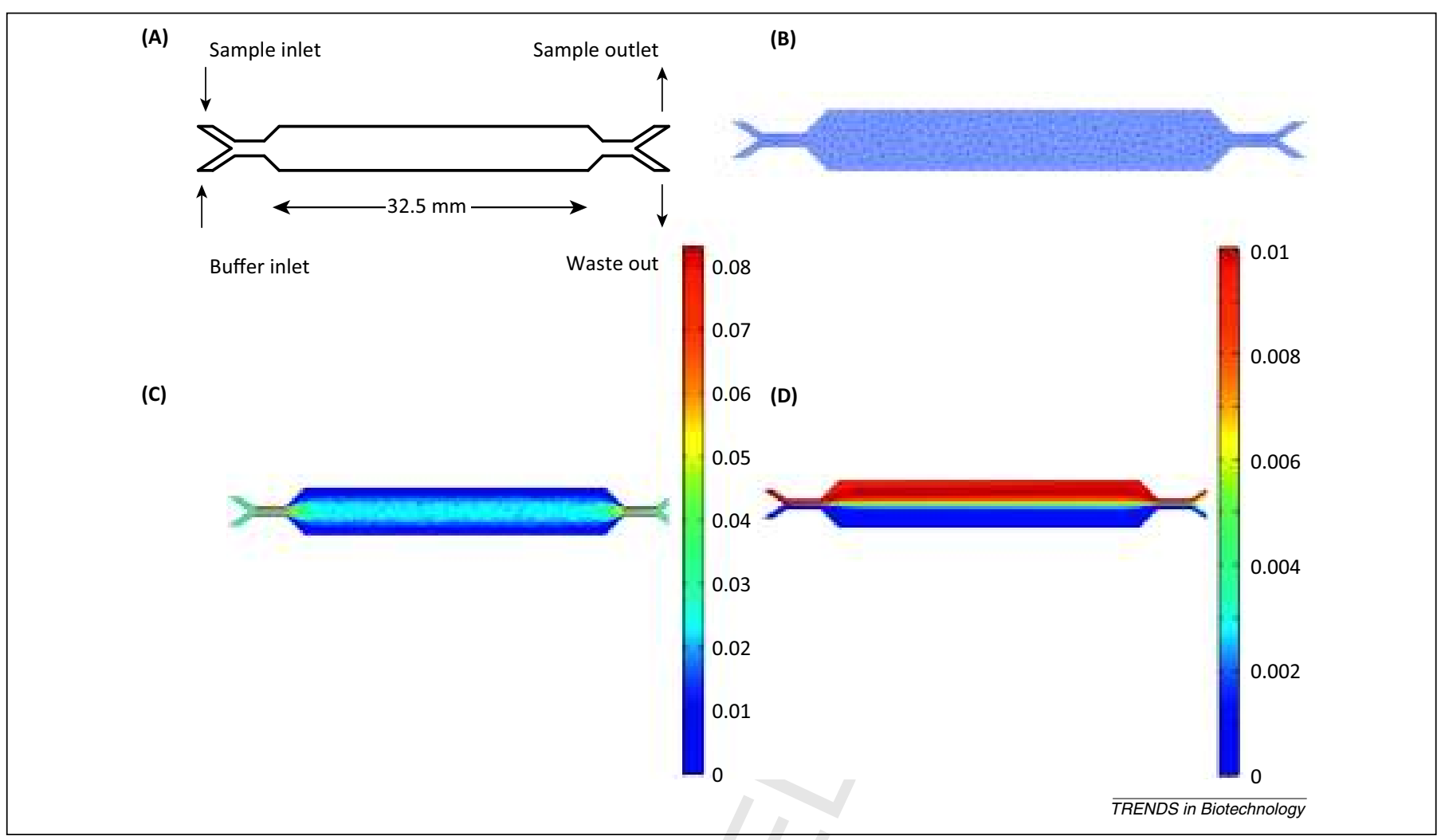

Figure 1. The different stages of model building and post-processing of the results. A simulation was carried out by considering a typical pressure gradient-pumped lab-on-

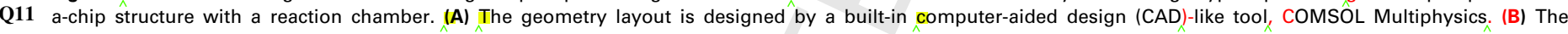
discretized domain is designed by the unmapped Delaunay triangulation method. (C) During post-processing of the obtained velocity field cell, the Reynolds number (Re) can be calculated and plotted (cell attribute means that the characteristic length in $R e$ is substituted by the average mesh size). (D) For better visibility, the resulting velocity field is traced by appropriate means and can be plotted in many different ways (a rainbow color plot is used in this instance).

\section{Single-phase Newtonian and non-Newtonian fluids}

In a simple case scenario, the streaming fluid in MBDs is assumed to be a single-phase Newtonian liquid [23]. In Newtonian fluids, the shear stress is proportional to the shear rate at constant temperature and pressure, and the proportionality is the dynamic viscosity. The NavierStokes equation

$\rho\left(\frac{\partial u}{\partial t}+(u \cdot \nabla) u\right)=\nabla\left(-p I+\eta\left(\nabla u+(\nabla u)^{T}\right)\right)$

of fluid motion is the generally used continuum mechanics model for describing the flow of incompressible fluids, which is usually coupled to the so-called continuity equation

$\nabla \cdot u=0$

where $u$ is the linear velocity, $\rho$ is the fluid density, $\eta$ is the fluid viscosity, $t$ is the time, and $p$ is the pressure. It is important to note thatEquation 2 describes the velocity flow field as a function of time, rather than the exact position of any part of it, that is, it treats the flow as a bulk. The calculated results can be visualized as trajectories of particles of the bulk phase, which helps in the interpretation of the results obtained. The governing equations can be solved in both steady-state and dynamic cases. Due to the assumption of a Newtonian flow, the dynamic viscosity of the streaming fluid (e.g., blood) is treated as constant and can be defined as $\eta=0.0035 \mathrm{~Pa}$ s [24]. In spite of simplicity, one-phase Newtonian models can be used in such complex problems as the design (shape and size) and optimization of electrospray ionization mass spectrometry (MS) coupling using microfluidic devices [25].

One of the most important applications of MBDs is cell sorting, that is, processing blood-like samples into separate cell types. However, in this instance, the Newtonian fluid assumption can be the origin of some inaccuracy. In MBDs, the typical convective velocity is $0.001 \mathrm{~m} / \mathrm{s}$ [26]; however, application of complex viscosity models is advised at velocities up to $0.2 \mathrm{~m} / \mathrm{s}$ [24]. Fortunately, different models, such as the Generalized Power law, the Walburn-Schneck, and the Carreau methods have been developed to describe the dynamic viscosity of streaming fluids as a function of the strain rate [24]. An innovative work was published in [12], where CFD simulations were used to determine the decrease of blood viscosity in the microchannels due to the Fahraeus effect, that is, when blood flows through a small diameter microchannel, the average hematocrit (solid particles of blood) in the microchannel is smaller than that in the reservoir so that blood viscosity decreases within the microchannel). Unfortunately, the estimated viscosity was not verified in an independent experiment.

Recently, reported single-phase models with the assumption of Newtonian fluid flow were applied to MBD modeling of cell capture processes. Jang and Wang [27] investigated a microfluidic device that was able to physically seize single carcinoma cells using a trap within the channel. The probability of successful cell capture was calculated from the percentage of the channel width where 
the velocity vectors flow into the trap. Also, in the work of Saias et al., a single-phase model with the same physical characteristics as water was used for optimizing the flow distribution in a microfluidic chamber [28].

\section{Multiphase modeling}

Although most published studies treated cells in the fluid sample as a continuous material (also referred to as continuum) [29,30] and the whole sample as a one-phase mixture of miscible liquids, some authors considered rare cell-containing flowing fluids as two-phase flow [31]. In the case of devices where a simple topology of channels (e.g., straight channel or $\mathrm{T}$ junction) was used without any barriers, single-phase continuum-based approaches resulted in realistic flow characteristics. However, in MBDs, where size-based cell capture was used or pillars were constructed at the inner chambers of the microchips in order to increase the specific surface area, it was necessary to take into account the size, volume, and shape of the flowing cells. Another technique described the nature of cells in the fluid flow by a two-phase system [32]. The authors simulated the cells as fluid with higher surface tension, which tended to minimize its surface area, thus, a spherical shape was obtained. The simulation suggested that the cells had an obstructive effect on the flow verifying the need for such a complex approach.

Hydrodynamic focusing is one of the basic functions of MBDs. It utilizes squeezing of the main stream at the intersections of two side streams and reshaping the original flow into a thin sheathed stream. Usually, focusing is carried out just in the horizontal plane, however, 3D stream converging has also been reported [33]. In this study, three phases, which could be miscible, were modeled using pure fluid sheath flows, while the main flow (the sample) had a solute as indicator of the width of the reshaped stream. In their model, a laminar flow characteristic was utilized in order to describe the hydrodynamic nature of the flows during focusing. Kitamori and coworkers fabricated a microfluidic chip for small-scale protein fractionation by isoelectric focusing. Although the authors did not do any modeling or simulation work, it is assumed that CFD could be an appropriate tool to investigate the fluid flow in their channel array [34].

An especially interesting and cutting-edge study was published by Hosseini and Tafreshi [35], dealing with particle flow simulation in a streaming fluid, which was essentially a two-phase problem. The pioneering aspect of this work was the ability to take into account the effect of instantaneous particle deposition on the barrier in the flow (filter fiber) and on each other. The authors enhanced features of the commercial CFD code with in-house developed subroutines. Their work shed light on the advantages of adding custom subroutines to common codes and indicated that available CFD programs Fluent and COMSOL Multiphysics, among others, can be utilized according to special needs by using external in-house written functions.

\section{Rare cell capture using different force fields}

In order to make classical, hydrodynamic flow-based MBDs more effective, different force fields can be applied such as electrokinetic (electrophoresis and electroosmosis), magnetic, or a combination of these. Furthermore, but without an analogy with electric or magnetic fields, it should be noted that an ultrasonic-induced pressure gradient can also be applied to lab-on-a-chip devices [36].

\section{Electric field-affected flows}

Electrokinetic focusing is one of the alternatives to hydrodynamic focusing, in which instead of pressure, electroosmotic flow (EOF) [37] is used for fluidic pumping. Beyond the typical plug shape of EOF, one of the most important advantages of electroosmotic focusing techniques is the lack of moving parts in the chip layout, reducing the risk of damage, while also decreasing cost. Kohlheyer et al. [37], Lin et al. [38], and Li et al. [39] used CFD simulations to analyze the distribution of the electric field inside a microfluidic chip. A recent publication [40] is especially interesting from the viewpoint of modeling and simulation of this phenomena. A simple equivalent (functional and constructional) electric circuit model was used in order to better understand the separation mechanism. This analogy-based simplification was possibly due to the flow profile of the streaming fluid in the microfluidic system.

Electric field-affected fluid flow can be modeled by solving the so-called modified Navier-Stokes equation

$\rho\left(\frac{\partial u}{\partial t}+(u \cdot \nabla) u\right)=\nabla\left(-p I+\eta\left(\nabla u+(\nabla u)^{T}\right)\right)+\rho_{e} E$

where $\rho_{e}$ is the volume charge density due to the presence of the electric double layer (also referred to as the Debye layer [40]) that can be defined as:

$\rho_{e}=-2 n_{0} e z \sinh e z \psi / k_{B} T$

where $e$ is the electron charge, $z$ is valence, $\psi$ is the electric potential of the Debye layer, $k_{B}$ is the Boltzmann constant, $n_{O}$ is the ion density of the bulk phase, and $T$ is temperature. It should be noted thatEquation 5 is a special solution [40] of the Poisson-Boltzmann equation, which generally describes the charge density of a double layer [41]. Please note that Equation 4 should be simultaneously solved with the continuity equation of Equation 3. Equation 4 assumes the existence of the electrical double layer on the channel walls, which induces EOF under applied electric field conditions. The relationship between the electric potential and the net charge density per unit volume is described by the Poisson equation. The electric double layer phenomenon has been intensively investigated (see Henderson and Boda [42] for a comprehensive review). Another relevant feature of the CFD approach is that the previously implemented governing equations (e.g., Equation 4) can be expanded and/or new partial differential equations can be derived. This equation-based approach is advantageous from the engineering viewpoint, because commonly available CFD softwares are numerical mathematical solvers rather than 'black box' easy-to-use tools.

Capillary electrophoresis (CE), one of the practical applications of the EOF phenomena in narrow bore tubes, is capable of rapid, high-resolution separation of very complex sample mixtures [43,44], utilizing the interplay between electrophoretic and electroosmotic velocities. 
Electric field-mediated separations are based on the hydrodynamic volume/charge ratio of the migrating species, making CE modeling complicated because the analyte molecules are subject to acidic dissociation as a function of the $\mathrm{pH}$ of the background electrolyte. As a first approximation, considering simple models, background electrolyte and analyte compounds are assumed to be completely ionized, so ion densities can be easily calculated and implemented as constants into the governing equation. However, it is more realistic if both the background electrolyte and analyte ions are considered to be only partially ionized. In spite of reliably measured data, acidic and basic dissociation constants of complex molecules like nucleic acids, amino acids/peptides, and glycans holding a charged tag can be estimated by computational methods [45]. COnductor-like Screening MOdel for Real Solvents (COSMO-RS) [46] is one of the most innovative ways to carry out calculations at the molecular level applying $a b$ initio quantum chemistry together with statistical thermodynamics.

It has been demonstrated [47] that computer-aided modeling and simulation can be utilized to find the optimal design for electrokinetic manipulation of fluidic movements in microfabricated modules such as cross-form, T-form, double T-form, variable-volume focused flow cross-form, and variable-volume triple-T-form. Exploiting the multiphysics ability of CFD approaches, that is, taking numerous physical phenomena into account at the same time, the principal transport mechanisms of electric fieldmediated flows can be described. Commonly applied theories $[47,48]$ apply the Poisson equation, the Nernst-Planck equation for calculation of ionic distribution concentration, the modified Navier-Stokes equation (Equation 4) together with the continuity equation (Equation 3), and a mass balance equation for diffusion and convection. Figure 2 depicts one possible implementation of the electrokinetic effect into the previously developed model example shown in Figure 1.

Dielectrophoresis (DEP) has been gaining interest recently among bio-analytical techniques as a manipulating tool for particles in solution [39]. The DEP phenomenon occurs when a dielectric (uncharged) particle or a cell is subject to a spatially nonuniform electric field [49]. DEP depends on the dielectric properties of the particles of interest and is fully controllable by varying the frequency and magnitude of the applied electric field. A typical computer modeling-based optimization was shown by Burgarella and coworkers [50] who investigated different electrode geometries in order to achieve the desired electrical field distribution and quantify the DEP force inside a microfabricated device. A parametric solver was used, which demonstrated that such models can indeed be useful tools for engineering optimization. In the course of parametric solution, one or more modeled parameters could be altered during the solution of the equation system in order to find the optimal value. Moreover, the authors prepared models for third-party software environments as imported objects or simulator engines. For example, COMSOL Multiphysics models can be exported to MATLAB as an $m$ script or a Java object. Detailed and clear mathematical formulae of
DEP simulations were derived in [51], where a $3 \mathrm{D}$ DEP-based focusing technique was studied. Another good example of CFD modeling was reported in [52] to assist in the understanding of unexpected separation phenomena by the simulation of electric field strength where particles with different dielectric properties were successfully fractionated resulting in $\geq 96.8 \%$ purity. Cell types of recent high interest, such as CTCs, could also have dielectric features different from those of the surrounding cells and other objects holding the promise to find new techniques for rare cell separation and capture.

\section{Magnetic field-affected flow}

While pressure and electrokinetic manipulation of fluidic flows in microchannels are usually referred to as label-free techniques, magnetophoretic isolation of species belongs to the so-called labeled methods [53]. Magnetophoretic techniques, also referred to as magnetic-activated sorting methods, have been thoroughly described for cells utilizing adhesion-based microfluidic cell-sorting devices in a recent review [54]. Magnetic-activated cell sorting (MACS) is a technique in which paramagnetic or superparamagnetic particles (e.g., beads) are used to improve the efficiency of cell sorting [55]. Particles, favorably monodisperse magnetic beads, are coated with antibodies with specific affinity to cell surface antigens of interest to catch targets. The applied magnetic field in the meantime retains the cellbead complexes from the fluidic flow, and the desired cells are yielded by decoupling the cells, for example, CTSs, from the complex.

A simple model, focusing on the collision and binding efficiency of cells and beads was published by Mohanty et $a l$. [26], where the cells and beads were treated as a continuum, their sedimentation was neglected, the fluid was assumed to be Newtonian, and the properties were considered the same as for water. The applied external magnetic field created by a magnetic dipole and the force acting on the beads were modeled by a user-defined function of the Fluent software package. Finally, the magnetophoretic phenomenon was implemented as an additional flux term in the transport equation. In spite of the simplicity of the model, it was appropriate to simulate a continuous immunomagnetophoretic cell sorter with an emphasis on binding kinetics.

A more complex and detailed simulation of rare cell capture using the immunomagnetic approach [56] took into account the non-Newtonian flow and the sedimentation of background red blood cells (RBCs) and rare target cells. The force acting on a given particle was the sum of (i) a pressure gradient, which moved the whole sample through the chip; (ii) sedimentation because of gravity; (iii) a drag force due to the magnetic field; and (iv) the viscous force contributed by the RBC content of the sample. The authors introduced a novel viscosity model, referred to as 'partial viscosity', which was a function of the volume $\mathrm{RBC}$ rate. The model allowed investigation of channel operational orientations, which was not routinely possible otherwise. The computational results were validated against experimental data, which justified the usage for such a complex approach. Another 
(A)

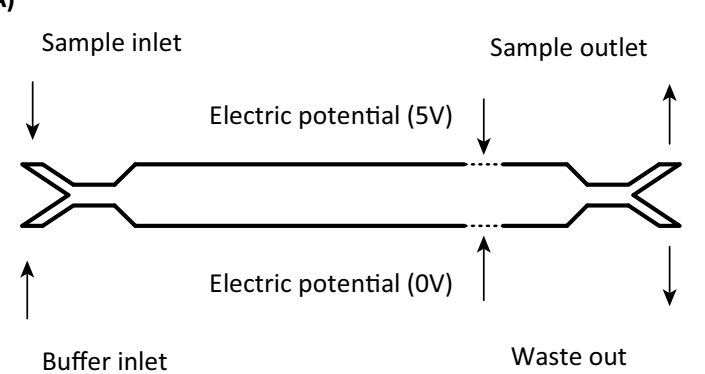

(C)

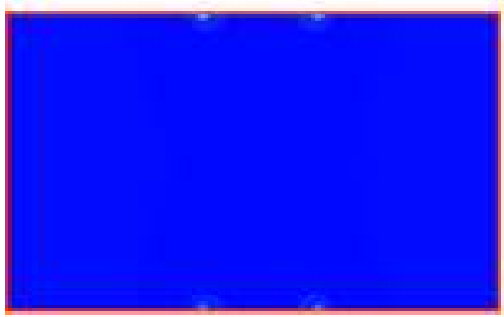

(B)
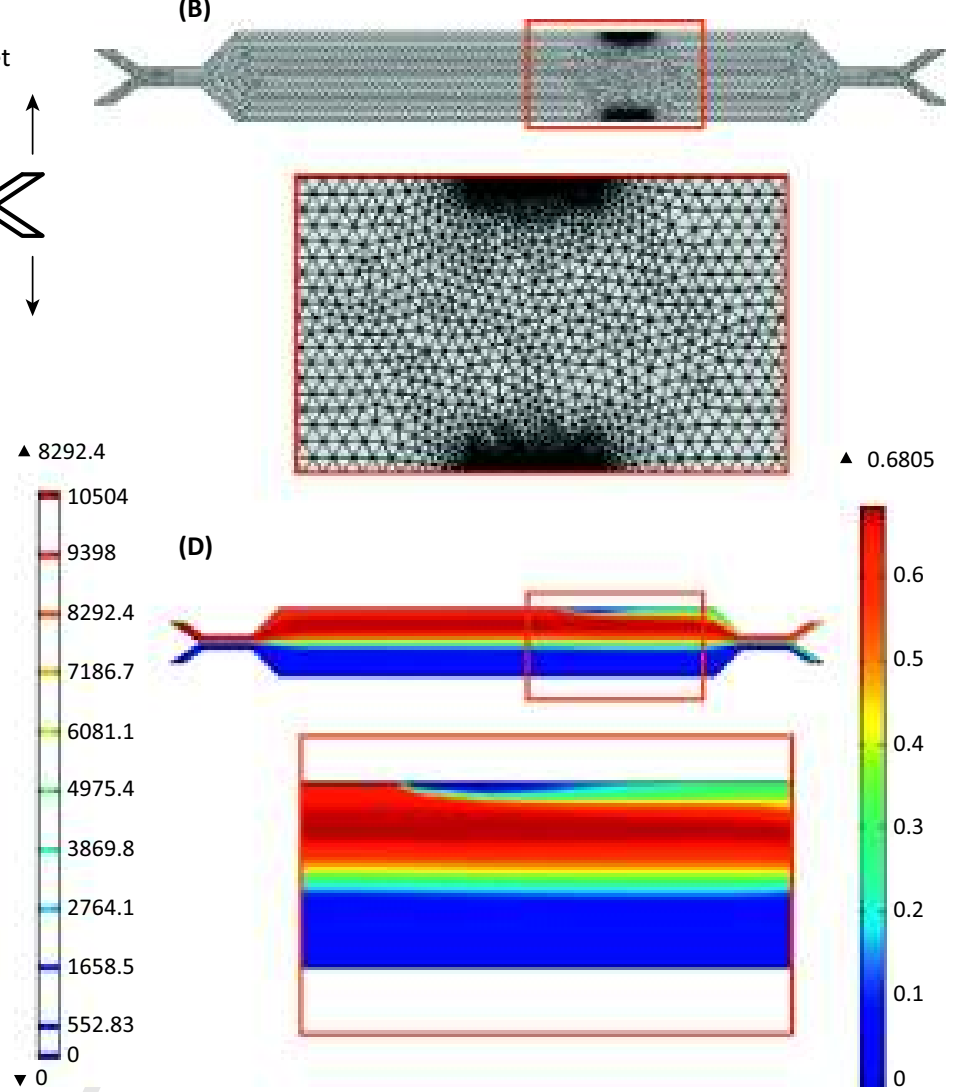

(D)

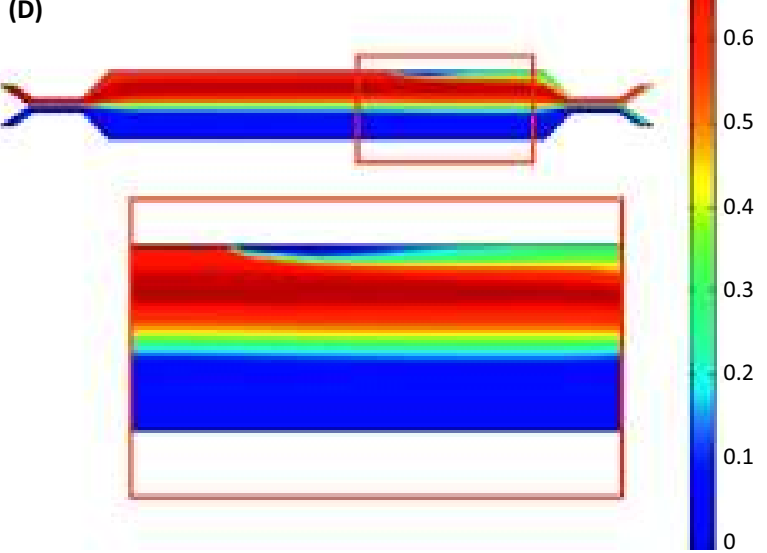

$\overline{T R E N D S ~ i n ~ B i o t e c h n o l o g y ~}$

Figure 2. The multiphysics approach. The simulation demonstration in Figure 1 was extended with a fictional electrokinetic effect. (A) The main geometry layout was not changed with the addition of two virtual electrodes with $5 \mathrm{~V}$ potential each (no spatial demand for the electrodes). (B) The refined mesh density was calculated at the critical points by using the Delaunay triangulation method; the results were in good agreement with the findings of [50]. (C) A contour plot of the obtained normalized electric field. (D) This panel clearly shows that the electrokinetic focusing diverted the normal flow (the velocity field was traced in a similar way to Figure 1D).

important practical application could be the calculation of shear stress exerted on the suspended cells during their flow through the microchannels modeled by CFD simulations $[15,57]$. In order to accurately estimate the induced stress, the discretization method was appropriately selected and tested [15].

\section{Concluding remarks and future prospects}

CFD modeling of MBDs covers a wide range of frequently used current state-of-the-art techniques. Modeling and simulation of MBDs are well developed, but more importantly, in addition to being considered as just a design tool, they can also be used during the interpretation of

\section{Box 2. Outstanding questions}

- Does CFD modeling and simulation of microfabricated biodevices represent feasible developmental support or simply provide an additional tool to verify the results?

- How can one describe the fluidic motion by numerical methods in such complex devices as lab-on-a-chip systems?

- Can rare cell capture be modeled by CFD with reliable accuracy with special emphasis on binding stringency/shear stress ratio of the cell-substrate interaction?

- Is it possible to model the streaming of blood-like samples, which behave like non-Newtonian fluids? Is the approach suitable to evaluate the dynamic viscosity of blood? experimental data. Deeper understanding of various physical phenomena by means of CFD may lead to engineering of more efficient MBDs (Box 2). Furthermore, modeling and simulation speeds up the development process of labon-a-chip devices, and also reduces their cost. In instances when samples contain only hundreds to several thousand cells, CFD-aided design of MBDs holds the promise of successful implementation. It is expected that in the near future, MBDs will likely be developed with the help of computational modeling, opening up new horizons in cellbased diagnostics, not only in the genomics field, but for proteomics, metabolomics, lipidomics, and glycomics studies as well.

Validation against experimental results is a common issue in all modeling and simulation approaches because the obtained data may not be reliable. Although velocity fields may be dependable in the case of simple geometries, they should be handled with care if the modeled geometry domain is complex or the multiphysics model is used. Computational modeling of rapidly changing phenomena and dynamically controlled effects are of particular interest and impose further challenges. We envision that comprehensive CFD modeling of MBDs will emerge in the future providing a particularly important toolset for cross-disciplinary research teams in the biotechnology, biomedical, and clinical diagnostics fields. 


\section{Acknowledgments}

The authors gratefully acknowledge the support of the Momentum grant \#97101 of the Hungarian Academy of Sciences (MTA-PE Translational Glycomics). The stimulating discussion with Dezso Boda on Chapter 4.1 Q9 [42] is also highly appreciated.

\section{References}

1 Autebert, J. et al. (2012) Microfluidic: an innovative tool for efficient cell sorting. Methods 57, 297-307

2 Gossett, D.R. et al. (2010) Label-free cell separation and sorting in microfluidic systems. Anal. Bioanal. Chem. 397, 3249-3267

3 Chen, J. et al. (2012) Microfluidic approaches for cancer cell detection, characterization, and separation. Lab Chip 12, 1753-1767

4 Lin, S.C. et al. (2012) Single channel layer, single sheath-flow inlet microfluidic flow cytometer with three-dimensional hydrodynamic focusing. Lab Chip 12, 3135-3141

5 Rozitsky, L. et al. (2013) Quantifying continuous-flow dielectrophoretic trapping of cells and micro-particles on micro-electrode array. Biomed. Microdevices 15, 859-865

6 Modak, N. et al. (2009) Cell separation in a microfluidic channel using magnetic microspheres. Microfluid. Nanofluid. 6, 647-660

7 Dutta, D. and Ramsey, J.M. (2011) A microfluidic device for performing pressure-driven separations. Lab Chip 11, 3081-3088

8 Horak, D. et al. (2013) Albumin-coated monodisperse magnetic poly(glycidyl methacrylate) microspheres with immobilized antibodies: application to the capture of epithelial cancer cells. J. Biomed. Mater. Res. A 101, 23-32

9 Wlodkowic, D. and Cooper, J.M. (2010) Microfabricated analytical systems for integrated cancer cytomics. Anal. Bioanal. Chem. 398, 193-209

10 Nagrath, S. et al. (2007) Isolation of rare circulating tumour cells in cancer patients by microchip technology. Nature 450, 1235-1239

11 Stott, S.L. et al. (2010) Isolation of circulating tumor cells using a microvortex-generating herringbone-chip. Proc. Natl. Acad. Sci. U.S.A. 107, 18392-18397

12 Kim, M. et al. (2010) A Microfluidic device for continuous white blood cell separation and lysis from whole blood. Artif. Organs 34, 996-1002

13 Chovan, T. and Guttman, A. (2002) Microfabricated devices in biotechnology and biochemical processing. Trends Biotechnol. 20, $116-122$

14 Khandurina, J. and Guttman, A. (2002) Bioanalysis in microfluidic devices. J. Chromatogr. A 943, 159-183

15 Didar, T.F. and Tabrizian, M. (2010) Adhesion based detection, sorting and enrichment of cells in microfluidic Lab-on-Chip devices. Lab Chip 10, 3043-3053

16 Weigl, B.H. et al. (2001) Lab-on-a-chip sample preparation using laminar fluid diffusion interfaces-computational fluid dynamics model results and fluidic verification experiments. Fresenius $J$. Anal. Chem. 371, 97-105

17 Ismagilov, R.F. et al. (2001) Pressure-driven laminar flow in tangential microchannels: an elastomeric microfluidic switch. Anal. Chem. 73, 4682-4687

18 Brody, J.P. et al. (1996) Biotechnology at low Reynolds numbers. Biophys. J. 71, 3430-3441

19 Stone, H.A. et al. (2004) Engineering flows in small devices. Annu. Rev. Fluid Mech. 36, 381-411

20 Erickson, D. (2005) Towards numerical prototyping of labs-on-chip: modeling for integrated microfluidic devices. Microfluid. Nanofluid. 1, 301-318

21 Hinsmann, P. et al. (2001) Design, simulation and application of a new micromixing device for time resolved infrared spectroscopy of chemical reactions in solution. Lab Chip 1, 16-21

22 Hangos, K. (2001) . In Process Modelling and Model Analysis (Hangos, K.M. and Cameron, I.T., eds), Academic Press

$23 \mathrm{Liu}, \mathrm{H}$. et al. (2012) Microfluidic biosynthesis of silver nanoparticles: effect of process parameters on size distribution. Chem. Eng. J. 209, $568-576$

24 Johnston, B.M. et al. (2004) Non-Newtonian blood flow in human right coronary arteries: steady state simulations. J. Biomech. 37, $709-720$
25 Grym, J. et al. (2006) Aerodynamic mass spectrometry interfacing of microdevices without electrospray tips. Lab Chip 6, 1306-1314

26 Mohanty, S. et al. (2010) Three-dimensional CFD modelling of a continuous immunomagnetophoretic cell capture in BioMEMs. Biochem. Eng. J. 51, 110-116

27 Jang, L.S. and Wang, M.H. (2007) Microfluidic device for cell capture and impedance measurement. Biomed. Microdevices 9, $737-743$

28 Saias, L. et al. (2011) Design, modeling and characterization of microfluidic architectures for high flow rate, small footprint microfluidic systems. Lab Chip 11, 822-832

29 Birnbaumer, G. et al. (2011) Rapid liposome quality assessment using a lab-on-a-chip. Lab Chip 11, 2753-2762

30 Xue, X. et al. (2012) Analysis of fluid separation in microfluidic T-channels. Appl. Math. Model. 36, 743-755

31 Mun, B. et al. (2010) Continuous cell cross over and lysis in a microfluidic device. Microfluid. Nanofluid. 8, 695-701

32 Chen, C.C. et al. (2004) Design and operation of a microfluidic sorter for Drosophila embryos. Sens. Actuat. B: Chem. 102, 59-66

$33 \mathrm{Kim}$, D.S. et al. (2009) An efficient 3-dimensional hydrodynamic focusing microfluidic device by means of locally increased aspect ratio. Microelectron. Eng. 86, 1343-1346

34 Ishibashi, R. et al. (2010) Two-step perpendicular free-solution isoelectric focusing in a microchamber array chip. Lab Chip 10, 2628-2631

35 Hosseini, S.A. and Vahedi Tafreshi, H. (2012) Modeling particle-loaded single fiber efficiency and fiber drag using ANSYS-Fluent CFD code. Comput. Fluids 66, 157-166

36 Antil, H. et al. (2012) Reduced order modeling based shape optimization of surface acoustic wave driven microfluidic biochips. Math. Comput. Simul. 82, 1986-2003

37 Kohlheyer, D. et al. (2008) A microfluidic device for array patterning by perpendicular electrokinetic focusing. Microfluid. Nanofluid. 4, $557-564$

38 Lin, Y.C. et al. (2004) Design and simulation of sample pinching utilizing microelectrodes in capillary electrophoresis microchips. Lab Chip 4, 60-64

$39 \mathrm{Li}$, M. et al. (2013) Improved concentration and separation of particles in a 3D dielectrophoretic chip integrating focusing, aligning and trapping. Microfluid. Nanofluid. 14, 527-539

40 Zhigang, W. et al. (2007) Microfluidic continuous particle/cell separation via electroosmotic-flow-tuned hydrodynamic spreading. J. Micromech. Microeng. 17, 1992

41 Gur, Y.et al. (1978) On the electrical double layer theory. I. A numerical method for solving a generalized Poisson-Boltzmann equation. J. Colloid Interface Sci. 64, 326-332

42 Henderson, D. and Boda, D. (2009) Insights from theory and simulation on the electrical double layer. Phys. Chem. Chem. Phys. 11, 3822-3830

43 Jorgenson, J.W. and Lukacs, K.D. (1983) Capillary zone electrophoresis. Science 222, 266-272

44 Mittermayr, S. et al. (2008) Mobility modeling of peptides in capillary electrophoresis. Trends Anal. Chem. 27, 407-417

45 Delaney, J.S. (2005) Predicting aqueous solubility from structure. Drug Discov. Today 10, 289-295

46 Klamt, A. et al. (2010) COSMO-RS: an alternative to simulation for calculating thermodynamic properties of liquid mixtures. Annu. Rev. Chem. Biomol. Eng. 1, 101-122

47 Tsai, C.H. et al. (2005) Numerical simulation of electrokinetic injection techniques in capillary electrophoresis microchips. Electrophoresis 26, 674-686

48 Andreev, V.P. and Lisin, E.E. (1993) On the mathematical model of capillary electrophoresis. Chromatographia 37, 202-210

49 Krishnan, J.N. et al. (2009) Rapid microfluidic separation of magnetic beads through dielectrophoresis and magnetophoresis. Electrophoresis 30, 1457-1463

50 Burgarella, S. et al. (2010) A modular micro-fluidic platform for cells handling by dielectrophoresis. Microelectron. Eng. 87, 2124-2133

51 Jen, C.P. et al. (2011) Three-dimensional focusing of particles using negative dielectrophoretic force in a microfluidic chip with insulating microstructures and dual planar microelectrodes. Electrophoresis 32, $2428-2435$ 


\section{Review}

Trends in Biotechnology xxx xxxx, Vol. xxx, No. x

52 Choi, S. and Park, J.K. (2005) Microfluidic system for dielectrophoretic separation based on a trapezoidal electrode array. Lab Chip 5, 1161-1167

53 Talasaz, A.H. et al. (2009) Isolating highly enriched populations of circulating epithelial cells and other rare cells from blood using a magnetic sweeper device. Proc. Natl. Acad. Sci. U.S.A. 106, 3970-3975

54 Baier, T. et al. (2009) Modelling immunomagnetic cell capture in CFD. Microfluid. Nanofluid. 7, 205-216
55 Gil, M. et al. (2013) Sperm selection using magnetic activated cell sorting (MACS) in assisted reproduction: a systematic review and meta-analysis. J. Assist. Reprod. Genet. 30, 479-485

56 Hoshino, K. et al. (2012) Computational analysis of microfluidic immunomagnetic rare cell separation from a particulate blood flow. Anal. Chem. 84, 4292-4299

57 Wurm, M. and Zeng, A.P. (2012) Mechanical disruption of mammalian cells in a microfluidic system and its numerical analysis based on computational fluid dynamics. Lab Chip 12, 1071-1077 